\title{
Molecular structure of the copia-like retrotransposable element Yokozuna on the W chromosome of the silkworm, Bombyx mori
}

\author{
Fumi Ohbayashi ${ }^{1}$, Toru Shimada ${ }^{2}$, Toshiyuki Sugasaki ${ }^{1}$, \\ Shinya Kawai ${ }^{1}$, Kazuei Mita ${ }^{3}$, Toshikazu Oshiki ${ }^{1}$, \\ and Hiroaki Abe ${ }^{1, *}$ \\ ${ }^{1}$ Department of Biological Production, Faculty of Agriculture, Tokyo University of Agriculture and Technology, \\ Fuchu, Tokyo 183-8509, \\ ${ }^{2}$ Graduate School of Agricultural and Life Sciences, The University of Tokyo, \\ Bunkyo-ku, Tokyo 113-8657, and \\ ${ }^{3}$ Genome Research Group, National Institute of Radiological Sciences, Anagawa, \\ Inage-ku, Chiba, 263-8555, Japan
}

(Received 30 August 1998, accepted 14 December 1998)

\begin{abstract}
We discovered a novel retrotransposable element, designated Yokozuna, on the $\mathrm{W}$ chromosome of Bombyx mori. The size of this element is $4738 \mathrm{bp}$, including a 208bp long terminal repeat (LTR) on one side and a 183-bp LTR on the other. This retrotransposable element is flanked by a 5-bp target site duplication, TAATT. Yokozuna contains a single long open reading frame (ORF) and the whole deduced amino acid sequence of ORF reveals strong homology with copia of Drosophila. Moreover, an alignment analysis of the reverse transcriptase (RT) sequences suggested that the Yokozuna element is the first Bombyx retrotransposable element belonging to the Ty1-copia group. The number of Yokozuna per haploid genome is approximately four copies. Although Yokozuna was discovered on the W chromosome, it is not specific for the $\mathrm{W}$ chromosome.
\end{abstract}

\section{INTRODUCTION}

The sex chromosomes of the silkworm, Bombyx mori, are designated $\mathrm{ZW}$ (equivalent to $\mathrm{XY}$ ) for the female and $\mathrm{ZZ}$ (XX) for the male (Tanaka, 1916). Femaleness is determined by the presence of a single $\mathrm{W}$ chromosome irrespective of the number of autosomes or of $\mathrm{Z}$ chromosomes, as shown by the observation that even ZZW and ZZZW individuals in the triploid and tetraploid states, respectively, are normal females (Hasimoto, 1933). Therefore, it is presumed that the female-determining gene, $\mathrm{Fem}$, is present on the $\mathrm{W}$ chromosome of $B$. mori. Although 400 or more visible mutations have been placed on linkage maps in B. mori (Doira, 1992; Goldsmith, 1995), no gene for a morphological character has yet been found on the normal W chromosome. Therefore, finding a DNA sequence on the W chromosome would be an important breakthrough for further analysis of the $\mathrm{W}$ chromosome.

Recently, we identified five randomly amplified polymorphic DNAs (RAPDs) that are female specific. One of the five, designated Female-218 RAPD, is specific to the female on translocation-carrying $\mathrm{W}$ chromosomes of

\footnotetext{
* Corresponding author.
}

B. mori (Abe et al., 1995, 1996; Ohbayashi et al., 1996); three of the five, designated W-Kabuki, W-Samurai and W-Kamikaze RAPDs, are specific to the female on the "true" $\mathrm{W}$ chromosomes of B. mori; and one of the five, designated $\mathrm{W}-Y a m a t o$ RAPD, is specific to the female on the W chromosome of Bombyx mandarina (Abe et al., 1998b). A computer search for deduced amino acid sequences of W-Kabuki, W-Samurai, W-Kamikaze, and W-Yamato RAPDs revealed that all four amino acid sequences show homology to the previously reported amino acid sequences of retrotransposable elements (Abe et al., 1998b).

To further analyze the $\mathrm{W}$ chromosome of $B$. mori, we constructed a genomic DNA lambda phage library and picked the lambda phage clone containing the W-Kabuki RAPD sequence by using a PCR strategy. We then sequenced the 18.1-kb DNA insert in which we discovered a novel copia-like long terminal repeat (LTR) retrotransposable element, designated Yokozuna. Here we describe the novel copia-like retrotransposable element, Yokozuna, from the $\mathrm{W}$ chromosome of $B$. mori.

\section{MATERIALS AND METHODS}

Insect. The $\mathrm{TW}+\mathrm{P}$ strain of $B$. mori was used in this 
study. TW $+\mathrm{P}$, which is congenic to the $\mathrm{J} 137\left(+^{p} /+^{p}\right)$ strain with respect to the $\mathrm{W}$ chromosome, was constructed by crossing a C137 (Dp $\left.[2 ; \mathrm{W}]+{ }^{p}, p / p\right)$ female with a male of the $\mathrm{J} 137$ strain and backcrossing the resulting $\mathrm{F}_{1}$ females to $\mathrm{J} 137$ males. $\quad+{ }^{p}$ represents the normal larval marking gene and $p$ (plain) represents the recessive allele; the $p / p$ larva is white. The TW+P strain has been maintained by repeated backcrossing of the females $\left(\mathrm{ZT}[\mathrm{W} ; 2]+{ }^{p}\right)$ to males of the J137 strain.

Genomic lambda phage library construction and screening. Two females of the $\mathrm{TW}+\mathrm{P}$ strain were used for genomic lambda phage library construction. Genomic DNA was extracted from larval posterior silk glands by the method of Bender et al. (1983). The DNA was further purified by phenol/chloroform extraction and was ethanol precipitated three times. The DNA was digested with Bam HI for $90 \mathrm{~min}$ and ligated into $\lambda$ EMBL3 (Stratagene). This ligation mix was packaged in vitro using a Gigapack II kit (Stratagene) according to the manufacturer's instructions, and plated onto Escherichia coli XL1-BlueMRA (P2).

We predicted that conventional screening of lambda phage libraries by hybridization would be difficult because the W-Kabuki RAPD sequence, based on a homology search, may contain two different elements. Thus, the resulting lambda phage library was screened by a locusspecific PCR strategy as described below. Initially, we determined the titer of the lambda phage library by serial dilution. In the initial round of screening, we made ten $9 \mathrm{~cm}$ plates, each containing nearly 10,000 plaques. Plates were removed from the incubator, covered with $8 \mathrm{ml}$ $\mathrm{SM}$ buffer, and stored at $4^{\circ} \mathrm{C}$ for $16 \mathrm{~h}$ to elute the lambda phage. Aliquots of $0.3 \mathrm{ml} \mathrm{SM}$ buffer containing phage particles from each plate were heated at $95^{\circ} \mathrm{C}$ for $5 \mathrm{~min}$ to elute lambda phage DNAs and then $1 \mu$ l was used as a template for PCR. The PCR protocol was that used by Abe et al. (1998a). To detect the W-Kabuki RAPD as a sequence characterized amplified region (SCAR) marker (Paran and Michelmore, 1993), PCR reactions with the Kabuki-1A primer (5'CCGCATCTACCTAACTCAGC-3') and Kabuki-2 primer (5'CGCATCTACCATACTGCTCA-3') consisted of 40 cycles: $94^{\circ} \mathrm{C}$ for $1 \mathrm{~min}, 50^{\circ} \mathrm{C}$ for $2 \mathrm{~min}, 72^{\circ} \mathrm{C}$ for $3 \mathrm{~min}$, and a final extension of $10 \mathrm{~min}$ at $72^{\circ} \mathrm{C}$. Female genomic DNA was amplified for positive controls. The SM buffer containing W-Kabuki was used in subsequent rounds. Similarly, during the second and third rounds of screening, we made ten plates containing nearly 1000 and 100 plaques, respectively. Finally, we made twenty plates, each containing nearly 20 plaques, and randomly picked 200 plaques by microspurtle and dropped each into $1 \mathrm{ml}$ of SM buffer. Three of 200 plaques were positive. One of these positive plaques, named K-1/3, was sequenced.

DNA sequencing and computer analysis. Standard DNA sequencing techniques were carried out according to
Sambrook et al. (1989). DNAs were sequenced using the Dye Terminator Cycle sequencing kit (Perkin Elmer ABI 377). The BLAST program (Altschul et al., 1990) was used to search for sequence similarities with known DNA (blastn) or protein (blastx) sequences. An amino acid sequence alignment was conducted with the CLUSTAL W (Thompson et al., 1994) and modified by visual inspection. A protein distance matrix was calculated from the aligned sequences (Fig. 4), where the insertions and deletions were ignored, by using Dayhoff's PAM amino acid substitution matrix (Dayhoff et al., 1978). A phylogenetic tree was constructed from this matrix using the Neighbor-Joining method (Saitou and Nei, 1987). From this, the tree in Figure 5 was constructed. To estimate confidence limits, 100 replicates of bootstrap analyses were performed using the programs of PHYLIP (Felsenstein, 1989). The nucleotide sequence of Yokozuna will appear in the DDBJ, EMBL, and GenBank nucleotide sequence databases with the accession number AB014676.

Construction of silkworm bacterial artificial chromosome library. A bacterial artificial chromosome (BAC) library for B. mori was constructed under collaboration with Dr. Pieter de Jong's laboratory (Osoegawa et al., 1998; Mita et al., unpublished). Silkworm high molecular DNA from the nuclei of posterior silk gland cells of 5th instar larvae of strain p50 was prepared by embedding it in agarose as described by Ioannou and de Jong (1996). The nuclei isolation of posterior silk gland cells was described previously (Ichimura et al., 1985). Preelectrophoresis and partial digestion of silkworm genomic DNA with EcoRI and size fractionation were carried out to isolate $150-200-\mathrm{kb}$ DNA fragments as described in the paper (Osoegawa et al., 1998). These fragments were ligated with EcoRIdigested and dephosphorylated pBACe3.6 vector. The conditions of ligation and transformation were also followed as described in the paper. The resultant BAC library of $B$. mori represents an estimated 11 -fold redundancy of coverage of the $B$. mori genome with an average insert size of more than $170 \mathrm{~kb}$.

Hybridization of BAC filter. The hybridization of a BAC high density replica (HDR) filter was carried out by the ECL hybridization method (Amersham). The hybridization conditions and labeling protocol were exactly followed per the manufacturer's directions.

Genomic Southern blots and hybridization. Genomic DNAs were prepared from posterior silk glands and digested with restriction endonucleases. Then, these DNA samples were electorophoresed in $1.0 \%$ agarose gels and blotted onto nitrocellulose filters (Biodyne plus membrane; Pall Biosupport Division $)$ in $20 \times \mathrm{SSC}(1 \times \mathrm{SSC}$ is $0.15 \mathrm{M} \mathrm{NaCl}$ plus $15 \mathrm{mM}$ sodium citrate) by capillary transfer, and were fixed by exposing to UV light. The hybridization solutions 
contained $50 \%$ formamide, $6 \times \mathrm{SSC}, 0.5 \% \mathrm{SDS}$, and $100 \mu \mathrm{g} /$ $\mathrm{ml}$ denatured salmon testes DNA. The Yoko-1 (nt $342-$ nt 2595) was used as a probe labeled with $\left[\alpha-{ }^{32} \mathrm{P}\right] \mathrm{dCTP}$ (ICN) by the random priming method using Random Primer DNA Labeling Kit Version 2 (Takara). Hybridiza-

\section{$\mathrm{K}-\mathrm{N}-$ \\ $\mathrm{M}$ 무 1/3 K2}

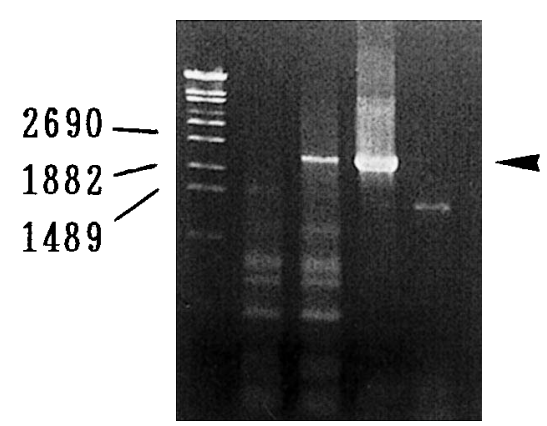

Fig. 1. Amplification patterns of genomic DNA from males and females of the $\mathrm{TW}+\mathrm{P}$ strain; the DNA from lambda clone $\mathrm{K}-1 / 3$, which contains the W-Kabuki RAPD sequence; and another lambda clone, N-K2, which lacks the W-Kabuki RAPD sequence. $\mathrm{M}$ : molecular size marker, $\lambda$-EcoT14 digest. The numbers at the left indicate base pairs. The arrowhead indicates the W-Kabuki RAPD amplified as a SCAR marker by using a set of primers, Kabuki-1A and Kabuki-2. tion was performed at $42^{\circ} \mathrm{C}$. The membrane was washed two times with $2 \times \mathrm{SSC}, 0.1 \%$ SDS for $10 \mathrm{~min}$ at $42^{\circ} \mathrm{C}$, and then for $15 \mathrm{~min}$ at $50^{\circ} \mathrm{C}$ under the same conditions.

\section{RESULTS}

Cloning of the Yokozuna element. We used a PCR strategy to pick a lambda phage clone, K-1/3, which contains the W-Kabuki RAPD sequence (Fig. 1) and we sequenced the DNA insert. This K-1/3 clone contained $18.1 \mathrm{~kb}$ insertional DNA. Based on a blastn and a blastx search, we determined that this 18.1 -kb insertional DNA is composed primarily of clusters of three retrotransposable elements, one retroposon, and one functionally unknown insertion. Each element, namely a non-LTR retrotransposable element BMC1 (Ogura et al., 1994), a retroposon $B m 1$ (Adams et al., 1986), functionally unknown inserted DNA (designated as FUI), and a novel copia-like LTR retrotransposable element, Yokozuna, was inserted into a novel gypsy-Ty3-like LTR retrotransposable element, Kabuki (unpublished) (Fig. 2). In the FUI, no significant ORFs were obtained, despite the presence of LTR-like sequences found at both ends.

Structure of Yokozuna LTR. The Yokozuna retrotransposable element was found to be $4738 \mathrm{bp}$ in length, with two LTRs (Fig. 3). The 5'- and 3'-LTRs were 208 bp

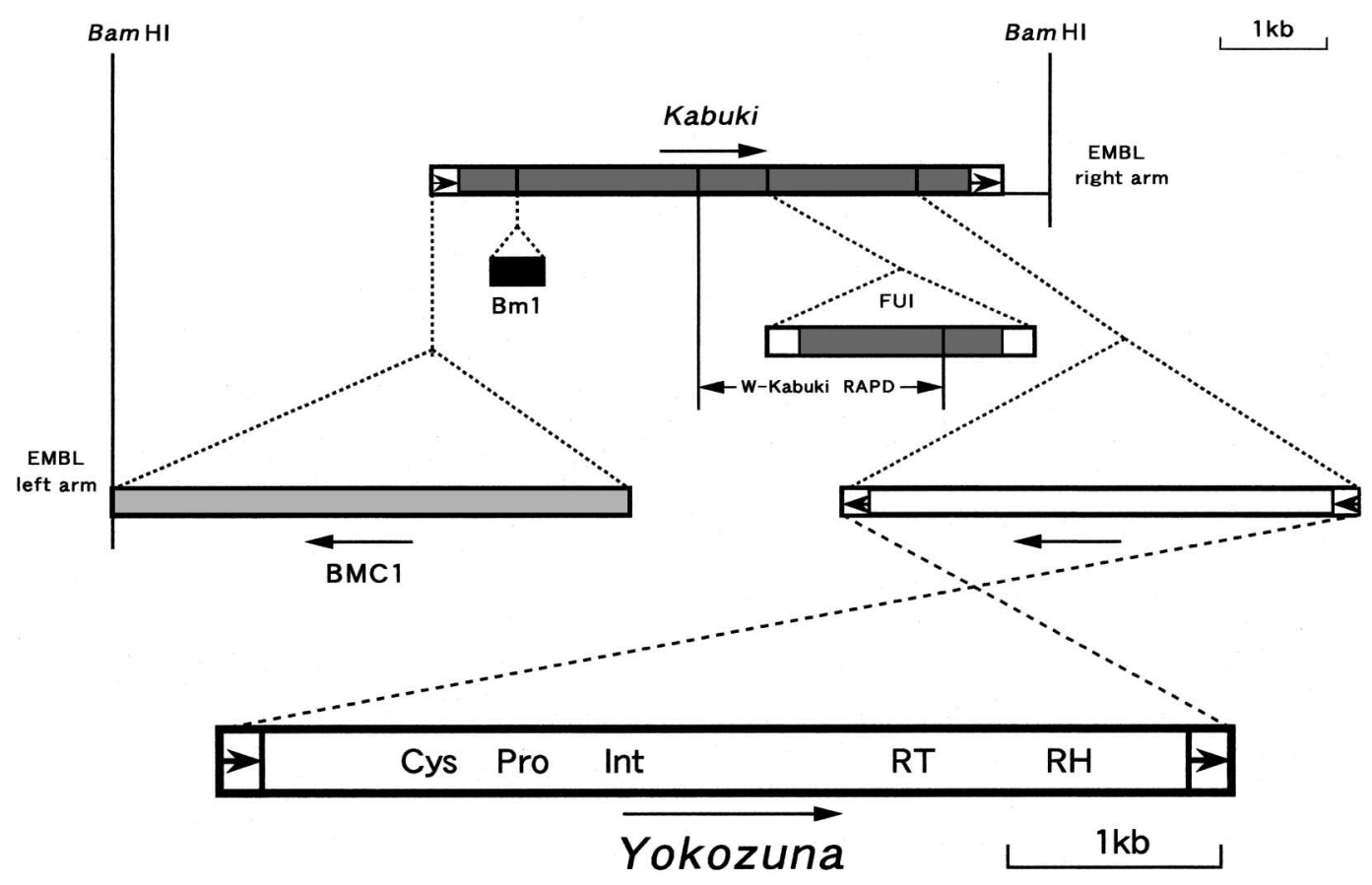

Fig. 2. Schematic view of the lambda clone K-1/3. This map is based upon DNA sequence information. Each block indicates a transposable element and each box with or without an arrow indicates one LTR or LTR-like sequence of each retrotransposable element. The Yokozuna element located in a novel LTR retrotransposable element Kabuki is shown flanked by its LTRs. The transcriptional orientation (arrows over or under each boxes) of the Yokozuna and BMC1 is opposite that of the Kabuki element. FUI, functionally unknown inserted DNA with two LTR-like sequences. The W-Kabuki RAPD sequence is extended from Kabuki to FUI. Restriction site, BamHI. Cys, cysteine and histidine motif; Pro, protease domain; Int, integrase domain; RT, reverse transcriptase domain; and RH, RNase H domain. 
caacataattTGTTGAGTATATTTTAGGATCTGTGCACTACTCTGTGGCTATATATGTTAGTQPATAA]TTAGTTTAGTTAGTCTATAATGTGTTATTACAAAATGTATCTATATCACTCATATAGTAACTCTATGATATCACTTCACTT CCCTTCGGCTCTGCTAATAAAATGGTGCCCGTACTTTTGTGTAATAATTTGCCAAGAGAAATCCAATACGCTTCCACTGCGCAACGACCGCCTCTTGGAGTTTAGAAGAATAGATTTAACGAAGAAGAAATATAATAGTAACAATAAAAG CGAAAAGTCAAGAGGAAGACAAAAAATACAAGAAAATTACTTGGAACTAAAATGGCGGACGACGCTTCAAGAAATTACTTCCAGAGTTTACTGGAACTAATTTCAGCACGTGGATATTTCGAATATCATGCATCCTAGAGGAAAGGGA M A D D A S R K L L P E F T G T N F S T W I F R I S C I L E E K E TGCAAAGAaGCAATTGAGGATGGCATAACAGAGGAAATATTGAAGTCAGAAAAATTCAAAAAGAAAGACGCAAAAGCTAGAaGTTTGATAGTCAACTGCCTCTCAGACAAACACTTGGAATACGTCAGAAGCGCTACGAGTGCAAAAGAG C K E A I E D G I T E E I L K S E K F K K K ATGATCGAAAACTTACGAAAAATATTCAAAGGAAAAAGTACGTTATCCGCGTTATACGTCAGGAAGAAATTATTAACCTTAAAATGTGACCCCAGTGTCGAACTGAGCGACCATTTCAACAATTTTGATATGTTAATAAGACAATTAGAA

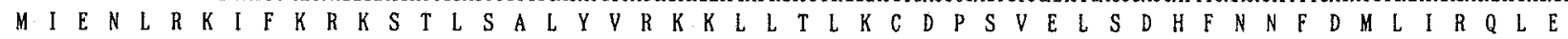
GAAACTGGAAGCACAGTAAATGAACAAGACAAGGTGTGCCACTTACTACTGACTATGCCTGACAGCTACAATACTACAATTACCGCCTTAGAAACTACTAATGTGGAACTTACAACAGAGTATGTCAAAAGCAAATTGTTAGATGCAGAA E T G S T V N E Q D $K$ K V C CTAAAAAACCAAAATAATTCAATGGAATCACAGGATGCACACAGCTTCCTCAGTTGCTTTAAATGTGGTAAAACTGGTCACAAAGCTATACAGTGCAGAACTGGACACTTAATGAGAGGACAGCATCGAGGCAAAATGGGCAACGTCGGA $L K N Q N N S M E S Q D A H S F$ TATTTCAACGGAaGTAACACTAGAGACTTCTATCGAGGCAACGGCAGAGACTTCAATCCAGGTAACGGCAGAGGCTTTAACCGAAGCAATCCCAGAGGCAAGGTGAGAGGACAATCGAGGGGATCATCAAGGGACACCATACTCCAACAA $\begin{array}{lllllllllllllllllllllllllllllllllllllllllllllllllll}Y & F & N & G & S & N & T & R & D & F & Y & R & G & N & G & R & D & F & N & P & G & N & G & R & G & F & N & R & S & N & P & R & G & N & V & R & G & Q & S & R & G & S & S & R & D & T & I & L & Q & Q\end{array}$ GGAAAAAGTGCTTTAAGTGAGAGAAATCCATTTTCTTTTGTTGCCAGTGAAAATGTACTATCTGTTGATGTGTATAATGAGGACATTAAGTTTATTATTGATTCAGGAGCATCTCAAAATTTAGTCATTGATAGTTATGAAAGTACATG G K S S A L L S E $R$ R N P F $F$ TCAAACATTGATTATTTAGAACTAAAACTAAGATTTATGTTGCTAATGGTCAATATTTGTTTTCAAGTAAAAAAGGGATTTTAAATGTAAATATAATAACTTACCTATAAAAATAGAAGCACTACTTGTCAAGGGGCTATCCCACAAC $\begin{array}{lllllllllllllllllllllllllllllllllllllllllllllllllll}S & N & I & E & Y & L & E & T & K & T & K & I & Y & V & A & N & G & Q & Y & L & F & S & S & K & K & G & I & L & N & V & K & Y & N & N & L & P & I & K & I & E & A & L & L & V & K & G & L & S & H & N\end{array}$ ATATTATCTGTTAAAAATTATTAGAGAAAGGAAATTCTGTCAGATTTCATAAAAATTCTGTATCAATTGCTAAAGGAAATAACATAATTTATGGTACCATGCTTAATAGTCTGTATGCAATCAATCTTACATTAAACTTAGAACAATGT

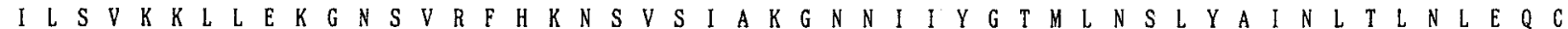
IATTCGATAAATAATGATAAGATCTATGGCATAAAAGGTTAGGACATGCTAATAGAAACACTTAAATTTATTGAAATTACCTATCTCAGAAAAACCATGTGGAATATGTGTAGAAGGAAAATCAACAGGATTACCATTCTCTACAACA Y S I N N D K D L

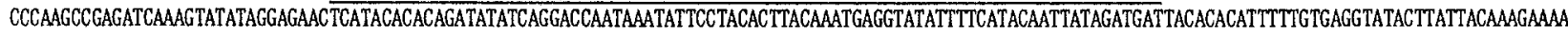

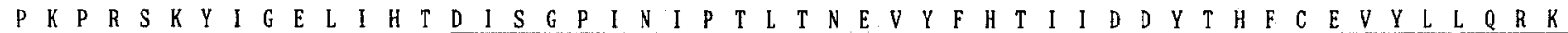
AGTGAGGCAACGGATAGGCTCATAGAATATGTTAATAGAATGGAAGGACAAATAGAATGTAAAGTCAAGAAAATCAGAAGTGATAATGGTGGTGAGTTTAAAAATGAAAAACTAAATAAATTTTGCAAAGATAAGGGAATTCTTCAACAG $\begin{array}{lllllllllllllllllllllllllllllllllllllllllllllllllll}S & E & A & T & D & R & L & I & E & Y & V & N & R & M & E & R & Q & I & E & C & K & V & K & K & I & R & S & D & N & G & G & E & F & K & N & E & K & L & N & K & F & C & K & D & K & G & I & L & Q & Q\end{array}$ TTTACTTTGCCTTATAGCCCACAGTCAAACGGAGTTTCAGAACGCATGAACAGAAATATTTATACAGAGCTCGGACTTTATTAATTGAATCTGGTTTACCAAAAACCTTATGGGGTGAAGCGGTGCGATGTGCTGTCTATCAAACCAAC $F \quad T L P$ Y S P Q S N G V S E R M N R N I Y N R A R T L L I E S G L P K T L W G E A V R C A V Y Q T AGATGTCCATCGTCCAACTTTCAAACCCAGCAGAGAAAATGTTGGAAACAAAGACTTAACAAGACTTAGGATATTTGGATCAAAATCATGGGTTCACATCATACCAAAACAAGATAAACTTTTACAAGGAGCTAAAGAAATGAGACTC

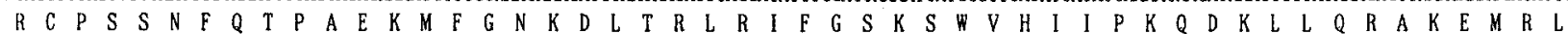
GTAGGATATAGCCCAAATGGATATCGTCTATGGGACCCTACAAGTAATAAAGTGATAGTGTCAAGAGATGTCAGAATTGATGAAACTCAATTAAACTACAAGGAAACTGAaGAaAaGCATAACATAAAAGGAGAACATACTATGAAGAT

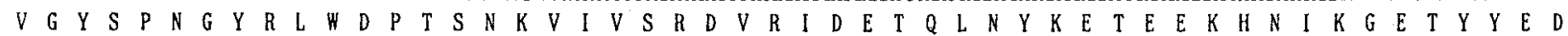
GAATTAATACAAAATGATGATACTGAGAaGGACACAGAaGGTGACCAAGAGATAAGAATACCTAATGAGAATGATGAAAACATGCAACATGATAGAATACCTGATAAAACAAATAAGAAGTATCAAAGATTATCAAGATGACGAAGAA

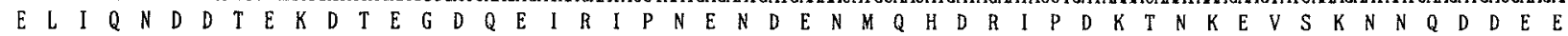

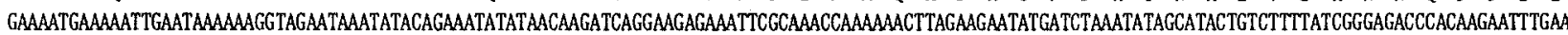

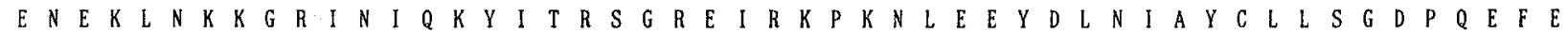
GATGCAATTCATGACAAAGACTGGGAAAAAGCAATAAAGACAGAATTAAATTCACAAGTGAAATTAGAGACATGGGAAGAAGCTACATTACCAATGGGAAGTAAAGCAATAGACACAAAATGGGTATTCAGAACAAAACAAAATGGAACC

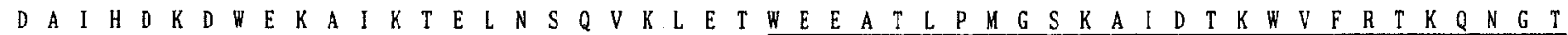
AAGAGAGCTCGATTAGTAGCGAAAGGTTTTCAGCAACAGAACAATGATAATCATTATGCTCCGGTAGCCAAATTGTCAACTATCAGATTAATGATGAGTTTAGCAGTTCAACTGGATTTGTCATTAAAGCAATTGGACGTACCAACAGCC

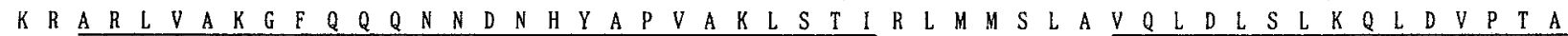

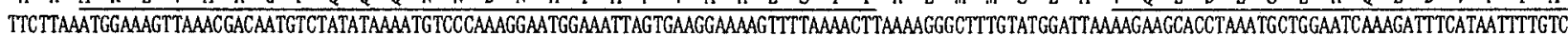
\begin{tabular}{lllllllllllllllllllllllllllllllllllllllllllllllllll}
$F$ & $L$ & $N$ & $G$ & $K$ & $L$ & $N$ & $D$ & $N$ & $V$ & $Y$ & $I$ & $K$ & $C$ & $P$ & $K$ & $G$ & $M$ & $E$ & $I$ & $S$ & $E$ & $G$ & $K$ & $V$ & $L$ & $K$ & $L$ & $K$ & $R$ & $A$ & $L$ & $Y$ & $G$ & $L$ & $K$ & $E$ & $A$ & $P$ & $K$ & $C$ & $W$ & $N$ & $Q$ & $R$ & $F$ & $H$ & $N$ & $E$ & $V$ \\
\hline
\end{tabular} ACTCAGAAAGGGTTTGTACAATCACAGCATGATTTATGTTTATATGGAAAAGGAAAAATTTGGATATTACTGTATGTGGATGATATTTTATACCTTGGAAATAGCAATGAGATGATAAAGGAATTAGAAAAGGAATTTAAGTAAAAAAC

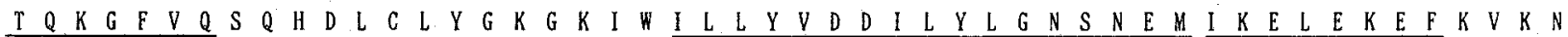
TTAGGAGAGGTACATCAATATTTAGGATTGGAAGTAACAAGAACAGAAGACAGCTTGGAAATAGGCAAACAGAAATAATTAAAGGCTTCTGGAGAAATATCACATGCAAGATTGCAAATCAAGCAAGACTCCAATGGAAATTAATTTT

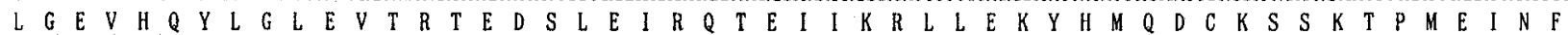

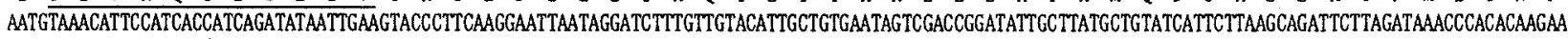
$\begin{array}{llllllllllllllllllllllllllllllllllllllllllllllllll} & V & N & I & P & S & P & S & D & I & I & E & V & P & F & K & E & L & I & G & S & L & L & Y & I & A & V & N & S & R & P & D & I & A & Y & A & V & S & F & L & S & R & F & L & D & K & P & T & Q & E\end{array}$

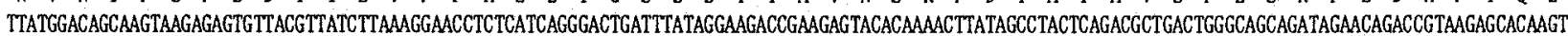

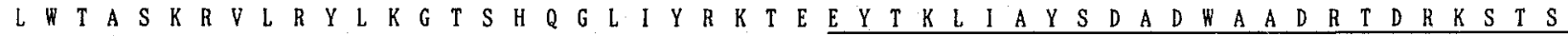
GGCTGTGCTATATATCAAGGCCAGAACTTAATAACTTGGTTCTCTAGAAAGCAAACATGTACCGCCCTGTCAACAGCTGAAGCTGAGTATGTGGCTGGAGCACACTCAATGAGTGAACTTATTCACATCAAAGGAATACTTTCTGACATA $\begin{array}{llllllllllllllllllllllllllllllllllllllllllllllllll}G & C & A & I & Y & Q & G & Q & N & L & I & T & W & F & S & R & K & Q & T & C & T & A & L & S & T & A & E & A & E & Y & V & A & G & A & H & S & M & S & E & L & I & H & 1 & K & G & I & L & S & D & I\end{array}$ CTAAGTACTAGTATCATTTCAGCTCATTTATTGATTGACAATCAGAGTACAATAAAATTGATACAAAATCAGATGAATACTAAGGCTTCTAAACATATTGAAATAAAATATCACTACATTAAAGATT TAATTTATAAGAAAATAGTGTCA $L$\begin{tabular}{llllllllllllllllllllllllllllllllllllllllllllllllll}
$L$ & $S$ & $T$ & $S$ & $I$ & $I$ & $S$ & $A$ & $H$ & $L$ & $L$ & $I$ & $D$ & $N$ & $Q$ & $S$ & $T$ & $I$ & $K$ & $L$ & $I$ & $Q$ & $N$ & $Q$ & $N$ & $N$ & $T$ & $K$ & $A$ & $S$ & $K$ & $H$ & $I$ & $E$ & $I$ & $K$ & $Y$ & $H$ & $Y$ & $I$ & $K$ & $D$ & $L$ & $I$ & $Y$ & $K$ & $K$ & $I$ & $V$ & $S$ \\
\hline
\end{tabular} ATCTCATATGTGCCTACAATGAAAATATCTCTGATATATTTACAAAATCTTCACTATTCAAAACATAATTATTTTTGTAGCAAGTTGAATTTAGTTTAAATATTGTATTAGAAAAGCTATGCATATGTTATACATATTTAATTGTTT I S Y V P T N E N I S D I F T K S S T I Q K H N Y F C S K L N L V *

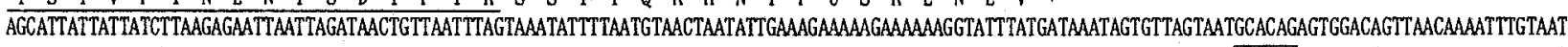
TCATTAAACTTATATTTTGITTGAACAATTTAAGCAGTTGTAAACTTCTATAAATTGAGGGGTGTGITGAGTATATTTTAGGATCTGTGCACTACTCTGTGGCTATATATGTTAGTCTATAATITIAGTTTAGTTAGTCTATAATGTGTT ATTACAAAATGTATCTATATCACTTCACTTCCCTTCCGCTCTGCIATAAAATGGTGCCCGTACTTTTGTGTAATAATTTGCCAAGAGAAATCCAATAtaattgaatg

140 290 440 33 590 83 740 133 890 183 1040 233 1190 283 1340 333 1490 383 1640 433 1790 483 1940 533 2090 583 2240 633 2390 683 2540 733 2590 783 2840 833 2990 883 3140 933 3290 983 3440 1033 3590 1083 3740 1133 3890 1183 4040 1233 4190 1283 4340 1316 4490 4640 4738

Fig. 3. Complete nucleotide sequence of the Yokozuna element from clone K-1/3. Lower case letters correspond to flanking sequences with the target site duplication double underlined. The LTRs begin and end with the underlined nucleotides TG and TA, respectively. The deduced amino acid residues of the single ORF are shown below the nucleotide sequence. Underlined amino acid residues correspond to conserved sequence motifs identified in other Ty1-copia group elements: one cysteine-histidine motif of gag-like genes, DSGA and ILS conserved motifs of proteases, $\mathrm{H}-\mathrm{X}_{4}-\mathrm{H}-\mathrm{X}_{\mathrm{N}}-\mathrm{C}-\mathrm{X}_{2}-\mathrm{C}$ and $\mathrm{D}, \mathrm{D}(35) \mathrm{E}$ motifs of integrases, the seven motifs of reverse transcriptase sequences, and a domain of RNase H from EYT to DIFTK. Sequences within the boxes correspond to the presumed promoter site (TATAAT) and poly(A) signal (AATAAA). The underlined nucleotide sequence adjacent to 3'-LTR is a polypurine tract. 
and $183 \mathrm{bp}$ in length, respectively, and were identical except for a deletion (or an insertion) of $25 \mathrm{bp}$. Since only one Yokozuna clone was sequenced, it remains to be determined which LTR length is major in Yokozuna. However, there was a presumed promoter site (5'-TATAAT-3') and a poly(A) signal (5'-AATAAA-3') in both LTRs. A region adjacent to the 5'-LTR seems likely to be a tRNA primerbinding site (PBS). However, data base screens for DNA sequence identities were not complementary to any tRNA. On the other hand, a region adjacent to the 3'-LTR of Yokozuna contained a short polypurine tract nine bp in length (8/9). LTRs of many retrotransposable elements have a short inverted repeat (5'-TG - CA-3') at the ends. However, the LTRs of Yokozuna differ from other retrotransposable element LTRs in that they do not terminate in CA.

ORF of Yokozuna. DNA sequencing of the Yokozuna element revealed a single ORF which occupies nearly the entire region between the LTRs. This ORF extends from position nt 342 to 4289 , starting with the amino acid (aa) initiation codon (methionine); the length of the ORF in the Yokozuna was 1316 amino acids (Fig. 3).

The Yokozuna ORF contained five regions with sequence similarity to functional domains found in the ORFs of the Ty1-copia group of LTR retrotransposable elements. The first domain was a putative nucleic acid binding domain (Covey, 1986). This domain of Yokozuna contained one putative cysteine and histidine motif $\left(\mathrm{C}-\mathrm{X}_{2}-\mathrm{C}-\mathrm{X}_{4}-\mathrm{H}-\mathrm{X}_{4}-\mathrm{C}\right)$ (Cys), located at aa 202, found in retroviral gag genes. The second domain was a potential protease domain (Pro) located immediately downstream of the gag-like domain. The conserved residues of this domain, "DSGA" followed by "ILS", are similar to those identified in copia (DSGA LMS) and TY912 (DSGA - LLS) (Doolittle, 1989). The third identifiable domain was an integrase (Int) located immediately downstream from the potential protease domain. An H-X $\mathrm{X}_{4}-\mathrm{H}-\mathrm{X}_{17}-\mathrm{C}-\mathrm{X}_{2}-\mathrm{C}$ motif seems to correspond to a conserved motif of retroviral integrase $\left(\mathrm{H}-\mathrm{X}_{3}-\mathrm{H}-\mathrm{X}_{22}-\mathrm{C}\right.$ $\mathrm{X}_{2}$-C) (Johnson et al., 1986). Also found was a highly conserved amino acid domain of integrase, referred to as $\mathrm{D}, \mathrm{D}(35) \mathrm{E}$, and composed of two invariant aspartate (D) residues and a glutamate (E) residue consistently separated by 35 amino acids (Kulkosky et al., 1992). The fourth domain was a potential reverse transcriptase (RT) domain, containing the seven motifs identified in all reverse transcriptase sequences (Xiong and Eickbush, 1988, 1990). The (final) fifth domain was a potential RNase H domain (RH) (Doolittle, 1989) located immediately downstream from the RT domain. The order of the five domains in the ORF of Yokozuna, Cys-Pro-Int-RT-RH, from 5 ' to 3', was identical to that within retrotransposons of the Ty1-copia group.

Copy number of Yokozuna in the genome. In a pre- liminary experiment, we characterized the BAC library with a BmE75 cDNA fragment, which was expected to correspond to a single copy gene, as a probe in the genome of $B$. mori. Nine positive clones were detected. Thus, this BAC library represented most of the $B$. mori genome at the expected redundancy of coverage. To estimate the copy number of Yokozuna in the genome, we performed Southern hybridization using BAC library filters and the Yoko-1 probe. Approximately 46 positive clones were detected in 36,864 clones. From this result, the number of Yokozuna per haploid genome was approximately four copies.

To estimate the difference of the copy number in the genome between male and female, we performed Southern blot hybridization using the Yoko-1 as a probe. The genomic hybridization pattern did not vary in either sex. This result indicates that Yokozuna is not specific to the $\mathrm{W}$ chromosome, but is present on the autosomes as well.

Phylogenic relationship of Yokozuna to other retrotransposable elements. A blastn search for DNA sequence similarity revealed that the DNA sequence of Yokozuna shows homology with many copia-like elements. For example, nt sequences 1822-2248 (Int domain), 30343347 (RT domain), and 4077-4336 (RH domain) exhibit nt identity of $54 \%$ with nt sequence $1837-2263,57 \%$ with nt 3178-3491 of Drosophila koepferae copia element DNA (GenBank accession No. X96971), and 53\% with nt $4422-$ 4681 of $D$. melanogaster copia element (accession No. M11240; Mount and Rubin, 1985), respectively (figure not shown).

A blastx search for deduced aa sequence similarity revealed that the aa sequence of Yokozuna ORF shows homology with Drosophila copias. For example, the highest level of homology was seen between the deduced aa sequence throughout the whole of the ORF of Yokozuna and the aa sequence of copia from D. melanogaster (accession No. M11240). The identity (similarity) between the two deduced aa sequences was calculated to be $30.8 \%$ $(57.5 \%)$ when a gap was counted as one substitution (figure not shown). A phylogenetic tree of retrotransposable elements has been constructed by comparison of deduced amino acid sequences of the RT domain (Xiong and Eickbush, 1990). The amino acid sequence of the RT domain of Yokozuna was compared to those of Ty1-copia group elements and the gypsy element (Fig. 4). Amino acid alignment revealed amino acid identities (similarities) of $35.0 \%$ (65.5\%), 32.2\% (59.6\%), 37.7\% (64.7\%), 34.9\% (63.6\%), and 18.3\% (45.1\%), with copia, 1731, Tnt1, Ta1, and $T y 1$, respectively (Fig. 4). The phylogenetic tree in Figure 5 indicates that Yokozuna is comparatively closer to the copia family than to Ty1. Figure 5 suggests that Yokozuna, copia, 1731, and Tnt1/Ta1 all diverged during a relatively short time, and have since been evolving independently over a long period, being that they are equally 


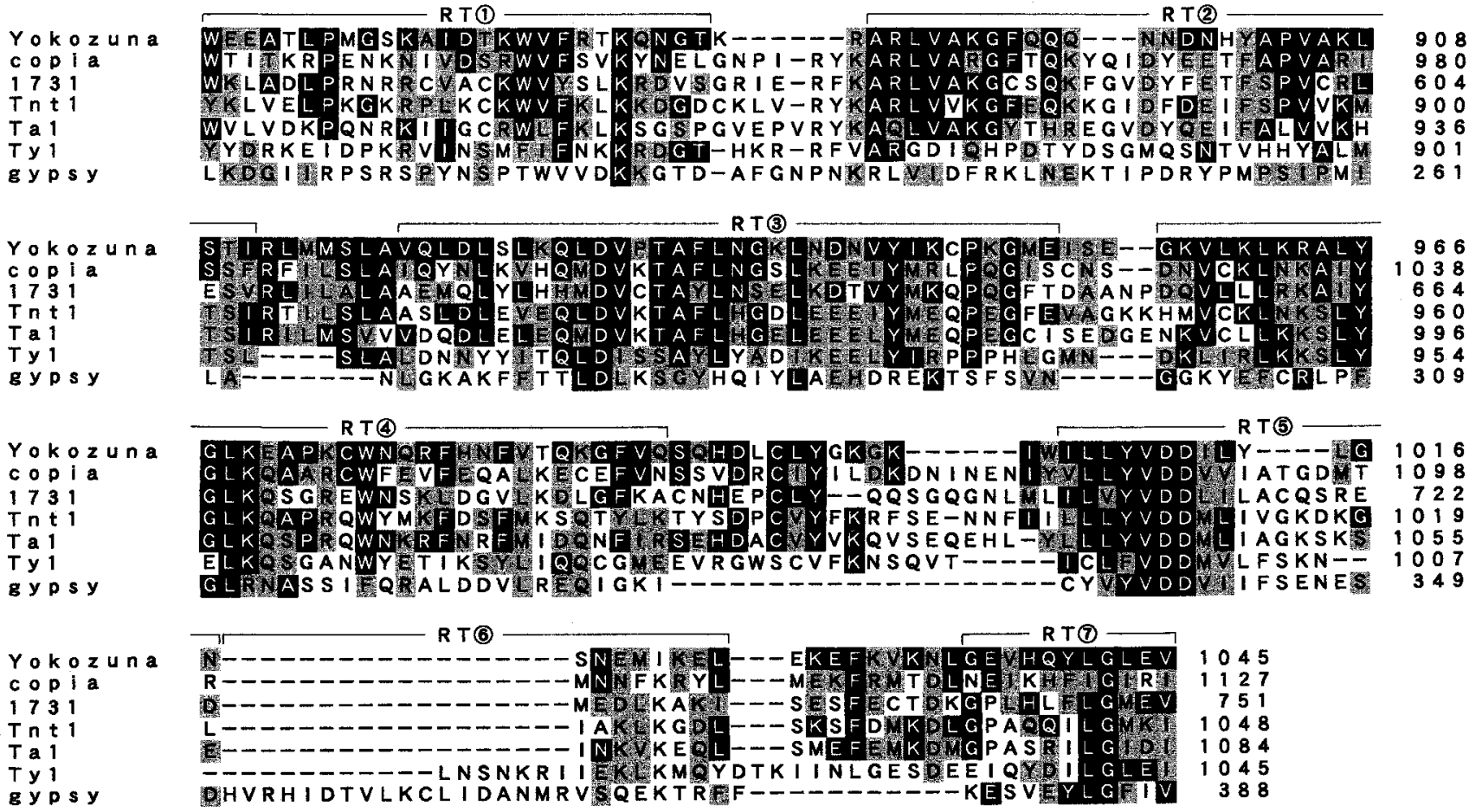

Fig. 4. Amino acid sequence alignment of RT-related sequences of the Yokozuna, other Ty1-copia group elements and gypsy elements. This figure was generated by performing a CLUSTAL W alignment and modified by visual inspection based upon Xiong and Eickbush (1990). Identical residues that are present in at least two of the seven amino acid sequences are highlighted by black boxes, while similar residues are highlighted by gray boxes. Numbers to the right indicate the number of residues from the $5^{\prime}$ end of the total ORF containing the RT sequences. Yokozuna, Yokozuna on the W chromosome of B. mori; copia and 1731, copia and 1731 of D. melanogaster (Mount and Rubin, 1985; Fourcade-Peronnet et al., 1988); Tnt1, Tnt1 of Nicotiana tabacum (Garvey et al., 1990); Ta1, Ta1 of Arabidopsis thaliana (Voytas and Ausubel, 1988); Ty1, Ty1 of Saccharomyces cerevisiae (Clare and Farabaugh, 1985); gypsy, gypsy of D. melanogaster (Marlor et al., 1986), and from RT (1) to RT (7) seven motifs identified in the RT sequence.

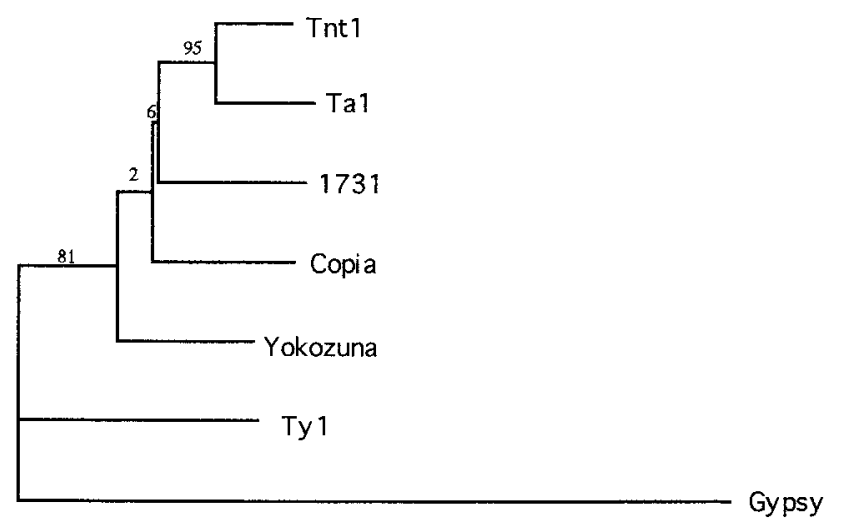

0.1

Fig. 5. Phylogenetic tree of the amino acid sequences of seven motifs in the RT domain of Yokozuna and other Ty1-copia group elements. The gypsy element from Drosophila melanogaster was used as an outgroup. The scale means corrected genetic divergences. The numbers above the branches are bootstrap values per 100 replicates. distant from each other.

\section{DISCUSSION}

We discovered that Yokozuna on the W chromosome of $B$. mori is a retrotransposable element belonging to the Ty1-copia group. The central region of this element contains a single ORF. This ORF contains a gag-like domain, a protease domain, an integrase domain, a reverse transcriptase domain, and an RNase $\mathrm{H}$ domain. Analysis of the DNA sequence and the deduced amino acid sequence of the ORF shows that Yokozuna is most similar to copia of Drosophila, which represents the Ty1-copia group. It remains to be investigated whether Yokozuna is active transcriptionally or transpositionally in vivo or in vitro and whether virus-like particles of Yokozuna are formed, as observed in D. copias (Shiba and Saigo, 1983; Yoshioka et al., 1992). Although Yokozuna was discovered on the $\mathrm{W}$ chromosome, it is not specific to the $\mathrm{W}$ chromosome.

All four aa sequences of the four RAPDs identified on the $\mathrm{W}$ chromosomes of $B$. mori and B. mandarina show homology to aa sequences of retrotransposable elements (Abe et al., 1998b). Furthermore, a complete full-length 
BMC1 retrotransposable element (Abe et al., 1998a), a retroposon Bm1 and a novel copia-like Yokozuna were tandemly inserted into a novel gypsy-Ty3-like retrotransposable element (unpublished). Thus, it is likely that many retrotransposable elements and their derivatives have accumulated on the $\mathrm{W}$ chromosome. Because crossing over is restricted to males in $B$. mori and because the $\mathrm{W}$ chromosome is transmitted only through the female line, the $\mathrm{W}$ chromosome is recombinationally isolated from the $\mathrm{Z}$ chromosome or the autosomes. The $\mathrm{W}$ chromosome would be expected to change (evolve) more slowly than other chromosomes and the inserted transposable elements would not likely be changed compared to other chromosomes.

Elements of the Ty1-copia group are present in a wide range of species from yeast (Sacchromyces), with the Ty1 elements, to Drosophila, with the copia elements, and include algae and many plants (Capy, 1998). Phylogenetic analysis has provided a body of data that suggests that horizontal transfer of the Ty1-copia group retrotransposons between different host species has occurred in the past (Flavell, 1993; Eickbush, 1995). Although it remains possible that significant levels of horizontal transfer of the Ty1-copia group retrotransposons occurs between species, in light of recent work it now seems unlikely (Flavell et al., 1997). Yokozuna, to our knowledge, seems to be the first full-length retrotransposable element identified belonging to the Ty1-copia group in Lepidoptera. It will be interesting to isolate Ty1-copia-like retrotransposable elements from a greater number of species in this family. It will be useful in consideration of horizontal transfer and evolution of the Ty1-copia group of retrotransposable elements.

This work was supported in part by CREST (Core Research for Evolutional Science and Technology) of Japan Science and Technology Corporation (JST).

\section{REFERENCES}

Abe, H., Shimada, T., Yokoyama, T., Oshiki, T., and Kobayashi, M. (1995) Identification of random amplified polymorphic DNA on the $\mathrm{W}$ chromosome of the Chinese 137 strain of the silkworm, Bombyx mori. J. Seric. Sci. Jpn. 64, 19-22. (In Japanese with English summary).

Abe, H., Ohbayshi, F., Shimada, T., Sugasaki, T., Kawai, S., and Oshiki, T. (1998a) A complete full-length non-LTR retrotransposon, $B M C 1$, on the $\mathrm{W}$ chromosome of silkworm, Bombyx mori. Genes Genet. Syst. 73, 353-358.

Abe, H., Shimada, T., Kawai, S., Ohbayashi, F., Harada, T., Yokoyama, T., Oshiki, T., and Kobayashi, M. (1996) Nucleotide sequence of the random amplified polymorphic DNA (RAPD) on the $\mathrm{W}$ chromosome of the silkworm, Bombyx mori (Lepidoptera: Bombycidae). Appl. Entomol. Zool. 31, 633637.

Abe, H., Kanehara, M., Terada, T., Ohbayashi, F., Shimada, T., Kawai, S., Suzuki, M., Sugasaki, T., and Oshiki, T. (1998b) Identification of novel random amplified polymorphic DNAs (RAPDs) on the W chromosome of the domesticated silkworm, Bombyx mori, and the wild silkworm, B. mandarina, and their retrotransposable element-related nucleotide sequences. Genes Genet. Syst. 73, 243-254.

Adams, D. S., Eickbush, T. H., Herrera, R. J., and Lizardi, M. (1986) A highly reiterated family of transcribed oligo (A)terminated, interspersed DNA elements in the genome of Bombyx mori. J. Mol. Biol. 187, 465-478.

Altschul, S. F., Gish, W., Miller, W., Myers, E. W., and Lipman, D. J. (1990) Basic local alignment search tool. J. Mol. Biol. 215, 403-410.

Bender, W., Spierer, P., and Hogness, D. S. (1983) Chromosome walking and jumping to isolate DNA from the Ace and rosy and bithorax complex in Drosophila melanogaster. J. Mol. Biol. 168, 17-33.

Capy, P. (1998) Phylogenies of transposable elements. In: Dynamics and Evolution of Transposable Elements (eds.: P. Capy, C. Bazin, D. Higuet, and T. Langin), pp. 103-156. Springer, Heidelberg.

Clare, J., and Farabaugh, P. (1985) Nucleotide sequence of a yeast Ty element: evidence for a novel mechanism of gene expression. Proc. Natl. Acad. Sci. USA 82, 2829-2833.

Covey, S. N. (1986) Amino acid sequence homology in gag region of reverse transcriptase elements and the coat protein gene of cauliflower mosaic virus. Nucleic Acids Res. 14, 623-633.

Dayhoff, M. O., Schwartz, R. M., and Orcutt, B. C. (1978) A model of evolutionary change in proteins. In: Atlas of Protein Sequence Structure. Vol. 5, Suppl. 3 (ed.: Dayhoff, M. O.), pp. 345-352, National Biomedical Research Foundation, Washington DC.

Doira, H. (1992) Genetical Stocks and Mutations of Bombyx mori. In: Important Genetic Resources (ed.: H. Doira), pp. 1-73. Silkworm Genetic Division, Institute of Genetic Resources, Fac. Agric. Kyushu Univ., Fukuoka, Japan.

Doolittle, R. F., Feng, D.-F., Johnson, M. S., and McClure, M. A. (1989) Origins and evolutionary relationships of retroviruses. Quart. Rev. Biol. 64, 1-30.

Eickbush, T. H. (1995) Mobile elements of lepidopteran genomes. In: Molecular Model Systems in the Lepidoptera (eds.: M. R. Goldsmith, and A. S. Wilkins), pp. 77-105. Cambridge Univ. Press, New York.

Felsenstein, J. (1989) PHYLIP-phylogeny inference package (version 3.2). Cladistics 5, 164-166.

Flavell, A. J. (1993) Ty1-copia group retrotransposons and the evolution of retroelements in the eukaryote. In: Transposable Elements and Evolution (eds.: J. F. McDonald), pp. 258269. Kluwer Academic Publ., Dordrecht, Netherlands.

Flavell, A. J., Pearce, S. R., Heslop-Harrison, P. (J. S.), and Kumar, A. (1997) The evolution of Ty1-copia group retrotransposons in eukaryote genome. In: Evolution and Impact of Transposable Element (ed.: P. Capy), pp. 185-195. Kluwer Academic Publ., Dordrecht, Netherlands.

Fourcade-Peronnet, F., D'Auriol, L., Becker, J., Bailibert, F., and Best-Belpomme, M. (1988) Primary structure and functional organization of Drosophila 1731 retrotransposon. Nucleic Acids Res. 16, 6113-6125.

Garvey, K. J., Oberste, M. S., Elser, J. E., Braun, M. J., and Gonda, M. A. (1990) Nucleotide sequence and genome organization of biologically active proviruses of the bovine immunodeficiencylike virus. Virology 175, 391-409.

Goldsmith, M. R. (1995) Genetics of the silkworm: revisiting an ancient model system. In: Molecular Model Systems in the Lepidoptera (eds.: M. R. Goldsmith, and A. S. Wilkins), pp. 21-76. Cambridge Univ. Press, New York.

Hasimoto, H. (1933) The role of the W-chromosome in the sex determination of Bombyx mori. Jpn. J. Genet. 8, 245-247. (In Japanese).

Ichimura, S., Mita, K., Zama, M., and Numata, M. (1985) Isola- 
tion of the giant ramified nuclei of the posterior silk glands of Bombyx mori. Insect Biochem. 15, 277-283.

Ioannou, P. A., and de Jong, P. J. (1996) Construction of bacterial artificial chromosome libraries using the modified P1 (PAC) system. In: Current Protocols in Human Genetics (eds.: N. C. Dracopoli, J. L. Haines, B. R. Korf, D. T. Moir, C. C. Morton, C. E. Seidman, J. G. Seidman, and D. R. Smith), pp. 5.15.1- 5.15.24, Wiley, New York.

Johnson, M. S. McClure, M. A. Feng, D.-F. Gray, J., and Doolittle, R. F. (1986) Assignment of enzymatic functions to specific sequences and homologies with nonviral enzymes. Proc. Natl. Acad. Sci. USA. 83, 7648-7652.

Kulkosky, J., Jones, K. S., Katz, R. A., Mack, J. P., and Skalka A. M. (1992) Residues critical for retroviral integrative recombination in a region that is highly conserved among retroviral/ retrotransposon integrases and bacterial insertion sequence transposases. Mol. Cell. Biol. 12, 2331-2338.

Marlor, R., Parkhurst, S., and Corces, V. (1986) The Drosophila melanogaster gypsy transposable element encodes putative gene products homologous to retroviral proteins. Mol. Cell. Biol. 6, 1129-1134.

Mount, S. M., and Rubin, G. M. (1985) Complete nucleotide sequence of the Drosophila transposable element copia: homology between copia and retroviral proteins. Mol. Cell. Biol. 5, 1630-1638.

Ogura, T., Okano, K., Tsuchida, K., Miyajima, N., Tanaka, H., Takada, N., Izumi, S., Tomino, S., and Maekawa, H. (1994) A defective non-LTR retrotransposon is dispersed throughout the genome of the silkworm, Bombyx mori. Chromosoma 103, 311-323.

Ohbayashi, F., Abe, H., Shimada, T., Kawai, S., Yokoyama, T., Oshiki, T., and Kobayashi, M. (1996) A commom random amplified polymorphic DNA in the silkworm, Bombyx mori is shared by $\mathrm{W}$ chromosomes onto which the normal marking, Sable and Black genes are translocated respectively. J. Seric. Sci. Jpn. 65, 395-398. (In Japanese).

Osoegawa, K., Woon, P. Y., Zhao, B., Frangen, E., Tateno, M., Catanase, J. J., and de Jong, P. J. (1998) An improved approach for construction of bacterial artificial chromosome libraries. Genomics 52, 1-8.

Paran, I., and Michelmore, R. W. (1993) Development of reliable PCR-based markers linked to downy mildew resistance genes in lettuce. Theor. Appl Genet. 85, 985-993.

Promboon, A., Shimada, T., Fujiwara, H., and Kobayashi, M. (1995) Linkage map of random amplified polymorphic DNAs (RAPDs) in the silkworm, Bombyx mori. Genet. Res. 66, 17.

Saitou, N., and Nei, M. (1987) The neighbor-joining method: a new method for reconstructing phylogenetic trees. Mol. Biol. Evol. 4, 406-425.

Sambrook, J., Frisch, E. F., and Maniatis, T. (1989) Molecular cloning: A Laboratory Manual. 2nd ed. Cold Spring Harbor Laboratory Press, Cold Spring Harbor, NY.

Shiba, T., and Saigo, K. (1983) Retrovirus-like particles containing RNA homologous to the transposable element copia in Drosophila melanogaster. Nature 302, 119-124.

Tanaka, Y. (1916) Genetic studies in the silkworm. J. Coll. Agric. Sapporo 6, 1-33.

Thompson, J. D., Higgins, D. G., and Gibson, T. J. (1994) CLUSTAL W: Improving the sensitivity of progressive multiple sequence alignment through sequence weighting, position-specific gap penalties and weigh matrix choice. Nucleic Acids Res. 22, 4673-4680.

Voytas, D. F., and Ausubel, F. M. (1988) A copia-like transposable element family in Arabidopsis thaliana. Nature 336, 242244.

Xiong, Y., and Eickbush, T. H. (1988) Similarity of reverse transcriptase-like sequences of viruses, transposable elements, and mitochondrial introns. Mol. Biol. Evol. 5, 675-690.

Xiong, Y., and Eickbush, T. H. (1990) Origin and evolution of retroelements based upon their reverse transcriptase sequences. EMBO J. 9, 3353-3362.

Yoshioka, K., Kanda, H., Takamatsu, N., Togashi, S., Kondo, S., Miyake, T., Sakaki, Y., and Shiba, T. (1992) Efficient amplification of Drosophila simulans copia directed by high-level reverse transcriptase activity associated with copia virus-like particles. Gene 120, 191-196. 